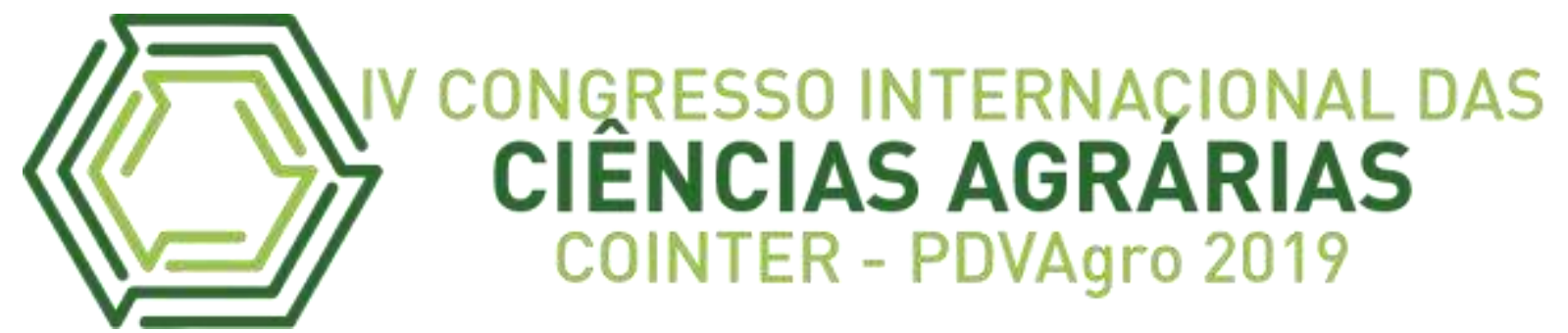

\title{
ANÁLISES FÍSICO-QUÍMICA E MICROBILÓGICAS DE FARINHA ELABORADA DO APROVEITAMENTO DA CASCA DE KIWI
}

\section{ANÁLISIS FÍSICO-QUÍMICO Y MICROBIOLÓGICO DE LA HARINA DE MEDICIÓN DE CÁSCARA DE KIWI}

\section{PHYSICAL-CHEMICAL AND MICROBIOLOGICAL ANALYSIS OF KIWI SHELL MEASURING FLOUR}

\author{
Apresentação: Comunicação Oral
}

\begin{abstract}
Newton Carlos Santos ${ }^{1}$; Sâmela Leal Barros ${ }^{2}$; Mylena Olga Pessoa Melo ${ }^{3}$; Amanda Priscila Silva Nascimento ${ }^{4}$; Josivanda Palmeira Gomes ${ }^{5}$
\end{abstract}

DOI: https://doi.org/10.31692/2526-7701.IVCOINTERPDVAgro.2019.0019

\section{Resumo}

O crescimento da agroindústria e a expansão da sua capacidade produtiva, provoca um aumento na quantidade de resíduos sólidos gerados nos processos produtivos. O reaproveitamento de partes não convencionais dos alimentos pode reduzir os gastos com a alimentação e os impactos ambientais do país, além de colaborar para a elaboração de novos produtos e matérias-primas. Nesse contexto, o presente trabalho teve como objetivo elaborar uma farinha, a partir do aproveitamento das cascas de kiwi, caracterizar físico-químicas e microbilogicamente, verificando assim, seus parâmetros microbiológicos. Para elaboração da farinha foram utilizadas cascas de kiwi cv. Hayward no qual foram, submetidas ao processo de secagem a $35^{\circ} \mathrm{C}$ no período de 72 horas. A farinha obtida foi analisada quanto aos seguintes parâmetros físico-químicos: umidade, sólidos totias, atividade de água, cinzas, lipídeos, proteínas, carboidratos, $\mathrm{pH}$, acidez, vitamina $\mathrm{C}$, clorofila a e b, clorofila total e carotenoides totais; os parâmetros microbiológicos avaliados foram: coliformes totais e termotolerantes, E. coli, bolores e leveduras, staphylococcus aureus, salmonela spp. O processo de secagem diminuiu a umidade, atividade de água da casca do kiwi, podendo ser armazenada e utilizada por um período de tempo mais longo, assim como este mesmo processo acarretou no aumento dos teores de cinzas, lipídeos, carboidratos e clorofilas evidenciando o potencial de aproveitamento deste resíduo. A partir dos resultados das análises microbiológicas, a farinha da casca de kiwi

\footnotetext{
1 Mestrando em Engenharia Agrícola, Universidade Federal de Campina Grande, UFCG, newtonquímicoindustrial@gmail.com

${ }^{2}$ Mestranda em Engenharia Agrícola, Universidade Federal de Campina Grande, UFCG, samelaleal7@gmail.com

${ }^{3}$ Mestranda em Engenharia e Gestão dos Recursos Naturais, Universidade Federal de Campina Grande, UFCG, mylenaopm@gmail.com

4 Doutoranda em Engenharia de Processos, Universidade Federal de Campina Grande, UFCG, amandapriscil@yahoo.com.br

${ }^{5}$ Dra. Professora Titular da Unidade Acadêmica de Engenharia Agrícola, Universidade Federal de Campina Grande, UFCG, josivanda@gmail.com
} 
apresentou resultados satisfatórios, dentro dos padrões estabelecidos pela legislação vigente, sendo considerada apropriada quanto à qualidade higiênico-sanitária. Estes resultados são importantes pois demostram que o processamento para elaboração da farinha atendeu aos requisitos necessários para sua futura utilização na fabricação de novos produtos alimentícios.

Palavras-Chave: Aproveitamento tecnológico, Clorofila, Resíduos agroindustriais

\section{Resumen}

El crecimiento de la agroindustria y la expansión de su capacidad productiva provoca un aumento en la cantidad de residuos sólidos generados en los procesos de producción. La reutilización de partes no convencionales de los alimentos puede reducir los costos de los alimentos y los impactos ambientales del país, y contribuir a la elaboración de nuevos productos y materias primas. En este contexto, el presente trabajo tuvo como objetivo elaborar una harina, a partir de la utilización de la cáscara de kiwi, para caracterizar fisicoquímica y microbilógicamente, verificando así sus parámetros microbiológicos. Para la preparación de la harina se utilizaron cáscaras de kiwi cv. Hayward en el que fueron sometidos al proceso de secado a $35{ }^{\circ} \mathrm{C}$ en 72 horas. La harina obtenida se analizó para los siguientes parámetros fisicoquímicos: humedad, sólidos totales, actividad del agua, cenizas, lípidos, proteínas, carbohidratos, $\mathrm{pH}$, acidez, vitamina $\mathrm{C}$, clorofila ayb, clorofila total y carotenoides totales; Los parámetros microbiológicos evaluados fueron: coliformes totales y termotolerantes, E. coli, moho y levadura, staphylococcus aureus, salmonella spp. El proceso de secado disminuyó la humedad, la actividad del agua de la cáscara de kiwi, se puede almacenar y usar durante un período de tiempo más largo, ya que este mismo proceso resultó en un mayor contenido de cenizas, lípidos, carbohidratos y clorofila que muestran el potencial uso de estos residuos. A partir de los resultados de los análisis microbiológicos, la harina de cáscara de kiwi presentó resultados satisfactorios, dentro de los estándares establecidos por la legislación vigente, siendo considerada apropiada para la calidad higiénico-sanitaria. Estos resultados son importantes porque demuestran que el procesamiento para la preparación de la harina cumplió con los requisitos para su uso futuro en la fabricación de nuevos productos alimenticios.

Palabras Clave: Clorofila, Residuos Agroindustriales, Uso Tecnológico

\section{Abstract}

The growth of agroindustry and the expansion of its productive capacity causes an increase in the amount of solid waste generated in the production processes. The reuse of non-conventional parts of food can reduce food costs and environmental impacts of the country, and contribute to the elaboration of new products and raw materials. In this context, the present work aimed to elaborate a flour, from the utilization of kiwi peel, to characterize physicochemical and microbilogically, thus verifying its microbiological parameters. For the preparation of the flour were used kiwi peel cv. Hayward in which they were subjected to the drying process at $35{ }^{\circ} \mathrm{C}$ within 72 hours. The obtained flour was analyzed for the following physicochemical parameters: moisture, total solids, water activity, ash, lipids, proteins, carbohydrates, $\mathrm{pH}$, acidity, vitamin $\mathrm{C}$, chlorophyll a and $\mathrm{b}$, total chlorophyll and total carotenoids; The microbiological parameters evaluated were: total and thermotolerant coliforms, E. coli, mold and yeast, staphylococcus aureus, salmonella spp. The drying process decreased the moisture, water activity of the kiwi peel, can be stored and used for a longer period of time, as this same process resulted in increased ash, lipid, carbohydrate and chlorophyll contents showing the potential use of this waste. From the results of the microbiological analyzes, the kiwi peel flour presented 
satisfactory results, within the standards established by the current legislation, being considered appropriate for the hygienic-sanitary quality. These results are important because they demonstrate that the processing for flour preparation met the requirements for its future use in the manufacture of new food products.

Keywords: Agroindustrial Waste, Chlorophyll, Technological Use

\section{Introdução}

O kiwi é uma fruta cítrica, originária da China e pertencente à Família Actinidiaceae e foi introduzida no Brasil apenas nos Anos 70, despertando grande interesse no mercado devido à alta produtividade e baixos custos de produção. Nutricionalmente, a fruta apresenta diversos nutrientes como o ácido ascórbico, beta-caroteno, fibras e potássio, estes nutrientes são vitais para o bom funcionamento do organismo humano e proporcionam a melhoria da pressão arterial, disfunções digestivas, estresse e depressão (SANTOS et al., 2019 a).

Com o crescimento do indústria de processamento de kiwi, houve também o aumento na geração de maior quantidade de resíduos, causando problemas econômicos e ambientais (GUO et al., 2018). Os resíduos de frutas (casca e semente) podem apresentar maior conteúdo nutricional do que as partes comestíveis. Além disso, esses resíduos podem conter compostos bioativos com maior capacidade antioxidante do que a polpa, dado que os perfis desses os fitoquímicos são diferenciados daqueles do outras partes da fruta). Durante o processamento de kiwi são gerados uma grande quantidade de resíduo sólido, observa-se também a redução no volume e diminuição na ocorrência de reações (químicas e microbiológicas), devido à retirada de água livre do produto e consequentemente ocorre a concentração de alguns componentes (WANG et al., 2018).

A secagem de cascas de frutas é uma excelente alternativa para o aproveitamento deste resíduo, pois agrega valor ao produto e diversifica seu uso. Pode-se utilizar a casca desidratada para a obtenção de farinha, que pode ser facilmente inserida na dieta através da inserção em produtos como bolos, biscoitos, massas e até mesmo bebidas isotônicas. No entanto, a secagem é um processo de transferência de calor e massa que deve ser bem entendido para alcançar a eficiência, desde perspectivas técnicas e econômicas (COSTA et al., 2016).

Nesse contexto, o presente estudo teve como objetivo elaborar uma farinha da casca de kiwi, proporcionando o aproveitamento deste resíduo agroindustrial e realizar a avaliação físico-química e microbiológica do produto final.

\section{Fundamentação Teórica}


O Brasil é um dos quarto maiores produtores de frutas no mundo, porém boa parte do que é produzido é desperdiçado durante as etapas pós-colheita, devido as deteriorações em frutas e verduras frescas e na geração de resíduos durante o processamento (LIMA et al., 2015).

O kiwi é o fruto proveniente de uma planta de videira perene que sofre desfolhação, contém altos níveis de antioxidantes benéficos, como vitamina $\mathrm{C}$, vitamina $\mathrm{E}$, carotenóides e compostos fenólicos. De maneira mais abundante estão presentes os polifenóis, que são importantes componentes pois apresentam propriedades antioxidantes, antivirais, antigênicas, antimicrobianas e anticancerígenas e são utilizados em o tratamento de doenças cardiovasculares (GUO et al., 2018).

O crescimento da agroindústria e a expansão da sua capacidade produtiva, provoca um aumento na quantidade de resíduos sólidos gerados nos processos produtivos. Estima-se que no Brasil são desperdiçados milhões de toneladas de alimentos e resíduos aproveitáveis por ano nas indústrias de alimentos. $\mathrm{O}$ aproveitamento destes resíduos provenientes do processamento de frutas e hortaliças (bagaço, casca, talos e sementes), podem ser utilizados como de diversos produtos. Quando comparados com a fruta in natura, estes resíduos apresentam quantidades superiores de nutrientes como fibras, minerais e compostos bioativos (CAMPOS et al., 2016; ARANHA et al., 2017).

Estes resíduos apresentam elevado potencial de aproveitamento, devido ao seu elevado valor nutricional, apresentando principalmente as fibras alimentares que atuam como marcador do estado geral de saúde e do cólon humano por meio do aumento do volume e maciez do bolo fecal, aumento da frequência e regularidade de evacuações, além de promover redução do colesterol e/ou glicose sanguíneos (LIMA et al., 2015).

A reutilização de não convencionais partes de alimentos para obtenção de farinhas, proporciona maior versatilidade destes produto que pode ser utilizado na elaboração de produtos de panificação e massas alimentícias, ampliando a diversidade de alimentos funcionais ofertados no mercado. Além disso, tem-se como vantagens a redução de custos de processamento e dos impactos ambientais (LIMA et al., 2015; SANTOS et al., 2019a).

A secagem de produtos alimentícios em estufa proporciona a redução do percentual de água livre no material, minimizando desta forma os efeitos de uma das principais variáveis responsáveis pela deterioração. A desidratação em estufa apresenta como vantagem também o baixo custo de operação e a elaboração de um novo produto (farinha) que apresenta segurança microbiológica adequada. Por isso, a secagem de alimentos é considerada como sendo um bom 
método de conservação, que apresenta como objetivo a remoção parcial de água dos alimentos (BENNEMANN et al., 2018).

Através da Resolução de nº 263, de 22 de setembro de 2005, a Agência Nacional de Vigilância Sanitária (ANVISA) regulamentou o processo produtivo de alimentos provenientes de cereais, amidos, farinhas e farelos. A Anvisa definiu que farinha é o termo que deve ser utilizado para os produtos obtidos de partes comestíveis de uma ou mais espécies de cereais, leguminosas, frutos, sementes, tubérculos e rizomas, que foram submetidos ao processo de moagem ou a processos tecnológicos que são considerados seguros para produção de alimentos, devendo ser denominadas utilizando o nome comum da espécie utilizada na elaboração (BRASIL, 2005).

A produção de farinhas a partir de resíduos alimentares possuem potencial para a elaboração de uma variedade de produtos como pães, bolos, biscoitos, bolachas, donuts, alimentos para o café da manhã (mingau instantâneo, produtos crocantes, tipo flocos), macarrão e molhos (molho de soja, ketchup). A qualidade funcional da farinha é importante para a determinação da sua utilidade em aplicações alimentícias. As condições de processamento (temperatura e trituração) influenciam diretamente nestas propriedades funcionais (OLATUNDE et al., 2015).

\section{Metodologia}

O trabalho foi desenvolvido no laboratório de Engenharia de Alimentos da Universidade Federal de Campina Grande, Campina Grande, Brasil. Os kiwis cv. Hayward (Actinidia deliciosa) utilizados foram adquiridos no mercado local da cidade de Campina Grande, Paraíba, Brasil.

A obtenção da farinha da casca do kiwi seguiu as etapas apresentadas no fluxograma da Figura 1. 


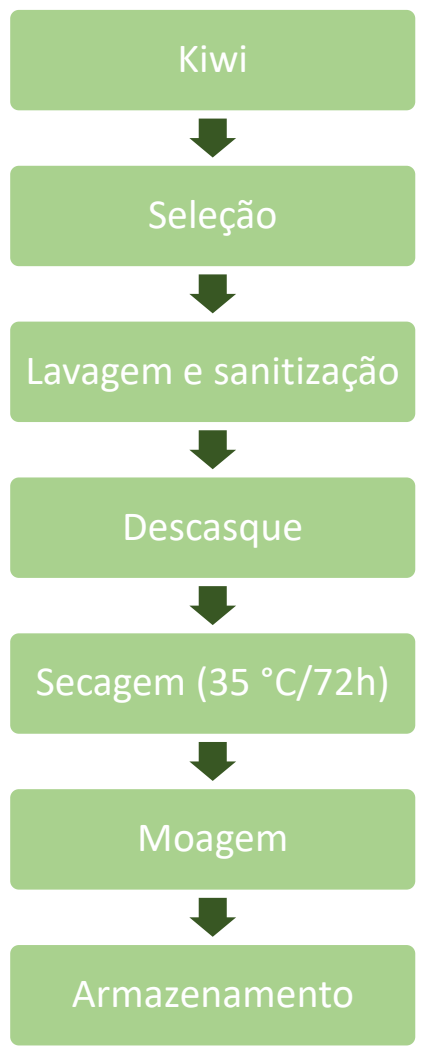

Figura 1: Fluxograma de etapas para obtenção da farinha da caca do kiwi. Fonte: Própria (2019).

Os kiwis foram selecionados, lavados em solução de hipoclorito de sódio a 200 ppm, por 15 min e, posteriormente, enxaguadas em água corrente. O descasque ocorreu de forma manual, com auxílio de uma faca doméstica.

A secagem da sua casca foi realizada em estufa de circulção de ar com velocidade do ar de $1,5 \mathrm{~m} . \mathrm{s}^{-1}$, na temperatura de $35^{\circ} \mathrm{C}$, durante $72 \mathrm{~h}$, nas quais as amostras foram distribuídas uniformemente em bandejas. Após a secagem, o produto foi submetido à operação unitária de moagem, fazendo-se uso de um moinho de facas (Fabricante BOTINI).

Após secagem e moagem, a farinha da casca do kiwi foi acondicionada em embalagens herméticas e mantida em temperatrura ambiente.

\section{Caracterizações físico-químicas}

Foram realizadas as caracterizações físico-químicas na casca in natura e desidratada: umidade, sólidos totais, cinzas, lipídeos, proteínas de acordo com a metodologia proposta pelo Instituto Adolfo Luttz (BRASIL, 2008); a atividade de água $\left(\mathrm{A}_{\mathrm{w}}\right)$ foi determinada usando o dispositivo Decagon ${ }^{\circledR}$ Aqualab CX-2T a $25{ }^{\circ} \mathrm{C}$; o teor total de carboidratos foi calculado por diferença para obter $100 \%$ da composição total (FAO, 2003). 
As demais determinações de $\mathrm{pH}$, acidez titulável, sendo os resultados expressos em ácido cítrico, teor de ácido áscorbico (Vitamina C), determinados de acordo com a metodologia proprotas pelo Instituto Adolfo Lutz (BRASIL, 2008); a derminação de clorofilas e carotenóides seguiram as metodologias propostas por Lichtenthaler (1987).

\section{Avaliação microbiológica}

Para a avaliação microbiológica da farinha da casca de kiwi, uma porção de $25 \mathrm{~g}$ do produto foi homogeneizada em $225 \mathrm{~g}$ de solução salina. A partir dessa diluição inicial, foram preparadas diluições seriadas utilizando o mesmo diluente.

A determinação de coliformes a $35^{\circ} \mathrm{C}$ (totais) e a $45^{\circ} \mathrm{C}$ (termotolerentes) foi realizada, através da determinação do número mais provável (NMP) de coliformes. Para a confirmação de E. coli foi utilizado o meio de cultura Ágar EMB, as placas foram inoculadas a partir dos tubos positivos de caldo EC em estufa a $35^{\circ} \mathrm{C}$ durante 24 horas.

Para a análise de Staphylococcus aureus, alíquotas de $0,1 \mathrm{~mL}$ foram transferidas para placas de Petri contendo ágar Manitol para semeadura em superfície. Após o semeio, as placas foram incubadas em temperatura de $36 \pm 1^{\circ} \mathrm{C}$ por $48 \mathrm{~h}$.

$\mathrm{Na}$ verificação de salmonella spp., uma porção de $25 \mathrm{~g}$ da amostra foi contida na água salina peptonada e incubada a $35^{\circ} \mathrm{C}$ por 24 horas. A amostra incubada foi transferida para placa de Petri e incubadas em temperatura $35^{\circ} \mathrm{C}$ por um período de 24 horas.

A análise de bolores e leveduras foi realizada através da técnica da semeadura em superfície, contendo ágar batata previamente acidificado com ácido tartárico, após o semeio as placas foram incubadas em temperatura ambiente por um período de 72h (BRASIL, 2003).

\section{Resultados e Discussão}

Na Tabela 1 constam os valores médios das caracterizações físico-químicas para a casca do kiwi (Actinidia deliciosa) in natura e desidratada convectivamente em estufa com circulação de ar. 
Tabela 1: Caracterização da casca de kiwi antes e após o processo de secagem.

\begin{tabular}{ccc}
\hline \multirow{2}{*}{ Parâmetros } & \multicolumn{2}{c}{ Cascas do kiwi $^{-}$} \\
\cline { 2 - 3 } In natura $^{1}$ & Desidratada $^{2}$ \\
\hline Umidade $(\mathrm{g} / 100 \mathrm{~g})$ & $84,17 \pm 0,07$ & $11,06 \pm 0,06$ \\
Sólidos totais $(\mathrm{g} / 100 \mathrm{~g})$ & $15,83 \pm 0,07$ & $88,94 \pm 0,06$ \\
Atividade de água $(\mathrm{Aw})$ & $0,983 \pm 0,003$ & $0,431 \pm 0,001$ \\
Cinzas $(\mathrm{g} / 100 \mathrm{~g})$ & $0,76 \pm 0,04$ & $4,04 \pm 0,07$ \\
Lipídeos $(\mathrm{g} / 100 \mathrm{~g})$ & $0,32 \pm 0,06$ & $2,15 \pm 0,26$ \\
Proteínas $(\mathrm{g} / 100 \mathrm{~g})$ & $4,8 \pm 0,10$ & $4,42 \pm 0,09$ \\
Carboidratos $(\mathrm{g} / 100 \mathrm{~g})$ & $9,95 \pm 0,11$ & $78,33 \pm 0,35$ \\
pH & $3,79 \pm 0,05$ & $4,03 \pm 0,15$ \\
Acidez $(\%$ ácido cítrico) & $0,248 \pm 0,001$ & $0,196 \pm 0,07$ \\
Vitamina C $(\mathrm{mg} / 100 \mathrm{~g})$ & $51,23 \pm 1,14$ & $49,04 \pm 1,82$ \\
Clorofila a $(\mathrm{mg} / 100 \mathrm{~g})$ & $0,145 \pm 0,038$ & $0,253 \pm 0,09$ \\
Clorofila b $(\mathrm{mg} / 100 \mathrm{~g})$ & $0,028 \pm 0,012$ & $0,088 \pm 0,04$ \\
Clorofila total $(\mathrm{mg} / 100 \mathrm{~g})$ & $0,173 \pm 0,016$ & $0,341 \pm 0,012$ \\
Carotenóides totais $(\mathrm{mg} / 100 \mathrm{~g})$ & $0,101 \pm 0,21$ & $0,291 \pm 0,37$ \\
\hline
\end{tabular}

Nota: ${ }^{1}$ base úmida; ${ }^{2}$ base seca.

Fonte: Própria (2019)

O teor de umidade obtido para a casca do kiwi in natura foi de $84,17 \mathrm{~g} / 100 \mathrm{~g}$, o processo de secagem ocasionou uma redução deste teor para $11,06 \mathrm{~g} / 100 \mathrm{~g}$, sendo este dento do valor máximo estipulado pela legislação (BRASIL, 2005) para farinhas, que é de 15,0 g/100g. Verificou-se ainda que a quantidade de sólidos totais foi maior para casca desidratada, tal crescimento é causado pela redução no teor de água. Santos et al. (2019b) obtiveram para casca da pitomba in natura teor de umidade de $66,50 \mathrm{~g} / 100 \mathrm{~g}$ e para a casca desidrata a $50{ }^{\circ} \mathrm{C}$ valor de $10,53 \mathrm{~g} / 100 \mathrm{~g}$, no entanto, para o teor de sólidos totais os valores foram 33,50 g/100g e 89,47 $\mathrm{g} / 100 \mathrm{~g}$, respectivamente.

Houve uma redução de $43,85 \%$ na atividade de água da casca in natura para a casca desidratada. Segundo Felows (2006) a atividade de água é um fator importante para o controle na taxa de deterioração do produto; geralmente, alimentos com atividade de água acima de 0,95 , conforme obtido para casca do kiwi in natura, são classificados como alimentos frescos altamente perecíveis, por isso tendem a se deteriorar rapidamente, fazendo-se necessária aplicação da secagem para reduzir este teor e aumentar sua conservação por mais tempo.

Observou-se aumento nos teores de cinzas, lipídeos e carboidaratos quando a casca do kiwi foi desidratada, exceto para o teor de proteínas que apresentou uma pequena degradação de 4,8 para 4,42 g/100g. Vasconcelos et al. (2019) ao obter e analisar a farinha do resíduo da 
goiaba (desidratada a $60{ }^{\circ} \mathrm{C}$ por $16 \mathrm{~h}$ ), obtiveram teor de cinzas de $1,68 \mathrm{~g} / 100 \mathrm{~g}$, sendo inferior a casca do kiwi do presente estudo. Valores inferiores de proteínas, foram observados por Garmus et al. (2009) na farinha da casca da batata inglesa $(2,5 \mathrm{~g} / 100 \mathrm{~g})$.

Também pode-se observar, um aumento do $\mathrm{pH}$ e redução da acidez das cascas desidratadas, fato este também observado por Lima et al. (2015) ao desidratarem a entrecasca da melancia para obtenção de farinha.

Uma pequena degradação da Vitamina $\mathrm{C}$ foi observada quando as cascas de kiwi foram submetidas ao processo de secagem, reduzindo em apenas 2,19 mg/100g. Segundo Rebouças et al. (2013) e Silva et al. (2018) as vitaminas são compostos muito sensíveis e podem ser degradadas por vários fatores, como temperatura, presença de oxigênio, luz, umidade, $\mathrm{pH}$, duração do tratamento a que o alimento foi submetido, entre outros.

Houve aumento da clorofila a, clorofila total e dos carotenóides totais, em relação aos teores obtidos para casca in natura (Tabela 2), no entanto, apenas o parâmetro de clorofila b, apresentou comportamento diferente, pois reduziu quando as cascas foram desidratadas. Foi observado por Santos et al. (2019c) que esses mesmos parâmetros apresentaram redução em relação ao quiabo in natura e liofilizado, no qual, obtiveram, respectivamente os seguintes teores de clorofila a $(14,21$ e 8,90 mg/100g), clorofila b $(25,44$ e 2,20 mg/100g), clorofila total $(38,56$ e $16,06 \mathrm{mg} / 100 \mathrm{~g})$ e carotenóides totais $(18,62$ e 3,43 mg/100g).

Os resultados das análises microbiológicas da farinha da casca do kiwi (coliformes a 35 ${ }^{\circ} \mathrm{C}$ e $45{ }^{\circ}$ C, E.coli, bolores e leveduras, Salmonella spp., Staphylococcus aureus) encontram-se na Tabela 2 .

Tabela 2: Avaliação microbiológica da farinha da casa de kiwi

\begin{tabular}{lc}
\hline Microrganismos & Valores médios \\
\hline Coliformes à $35^{\circ} \mathrm{C}(\mathrm{NMP} / \mathrm{g})$ & $<3,0$ \\
Coliformes à $45^{\circ} \mathrm{C}(\mathrm{NMP} / \mathrm{g})$ & $<3,0$ \\
Bolores e leveduras $(\mathrm{UFC} / \mathrm{g})$ & $1,2 \times 10^{2}$ \\
E. coli & Ausente \\
Staphylococcus aureus $(\mathrm{UFC} / \mathrm{g})$ & $1 \times 10^{1}$ \\
Salmonella spp. $(25 \mathrm{~g})$ & Ausência \\
\hline
\end{tabular}

Fonte: Própria (2019) 
Os resultados microbiológicos obtidos neste trabalho atenderam a legislação da ANVISA (Decreto n. ${ }^{\circ} 12.486$, de 20 de outubro de 1978), pois na contagem microbiológica do grupo dos coliformes, não se constatou nenhuma bactéria do grupo na farinha. Segundo Chisté et al. (2006), a presença de Coliformes é considerada como indicador de condições de higiene insatisfatórias na produção e/ou manipulação do alimento. O número elevado de Coliformes pode não significar contaminação direta com material fecal, mas sim manipulação inadequada, como higiene do manipulador, transporte e acondicionamento inadequados.

Santos et al. (2017) ao elaborarem e avaliarem farinhas obtidas a partir de cascas de jabuticaba, também não obtiverem presença de microrganismos do grupo coliformes.

Com relação aos bolores e leveduras o valor encontrado foi de $1,2 \times 10^{2} \mathrm{UFC} / \mathrm{g}$, portanto, considerado dentro do padrão, pois, conforme a ANVISA, o padrão máximo de bolores e leveduras é de $10^{3} \mathrm{UFC} / \mathrm{g}$. Indicando assim que, durante o processamento do kiwi para obtenção da farinha utilizaram-se boas práticas de fabricação.

A Salmonella spp. não apresentou nenhum crescimento na farinha atendendo a legislação vigente que é ausência em 25 g. Borges et al. (2009), ao trabalharem com a farinha obtida da banana verde também obtiveram ausência da Salmonella spp. A sua presença é potencialmente capaz de causar enfermidades e, portanto, tornando o alimento impróprio para o consumo humano.

Para a determinação de Staphylococcus aureus, houve a formação de 1 x $10^{1}$ Unidades Formadora de Colônias (UFC/g), uma vez que a legislação em vigência estabelece ausência em 0,1 g. A análise de Staphylococcus spp. serve para confirmar o envolvimento em surtos de intoxicação alimentar e ainda como indicador de contaminação pós-processo ou das condições de sanitização das superfícies destinadas ao contato com o alimento (ALVES e JARDIM, 2010).

\section{Conclusões}

O processo de secagem diminuiu a umidade, atividade de água da casca do kiwi, podendo ser armazenada e utilizada por um período de tempo mais longo, assim como este mesmo processo acarretou no aumento dos teores de cinzas, lipídeos, carboidratos e clorofilas evidenciando o potencial de aproveitamento deste resíduo. 
A partir dos resultados das análises microbiológicas, a farinha da casca de kiwi apresentou resultados satisfatórios, dentro dos padrões estabelecidos pela legislação vigente, sendo considerada apropriada quanto à qualidade higiênico-sanitária.

Estes resultados são importantes pois demostram que o processamento para elaboração da farinha atendeu aos requisitos necessários para sua futura utilização na fabricação de novos produtos alimentícios.

\section{Referências}

ALVES, P. T.; JARDIM, F. B. B. Análise microbiológica de cachorros-quentes comercializados na cidade de Uberaba, MG. Cadernos de Pós-Graduação da FAZU, v.1, 2010.

ARANHA, J. B.; NEGRI, T. C.; MARTIN, J. G. P.; SPOTO, M. H. F. Efeito da radiação gama nos parâmetros microbiológicos, físico-químicos e compostos fenólicos de farinha de resíduos de frutas durante armazenamento. Brazilian Journal of Food Technology, v. 20, p.1-10123, 2017.

BENNEMANN, G. D.; BOTELHO, R. V.; TORRES, Y. R.; CAMARGO, L. A.; KHALIL, N. M.; OLDONI, T. L.; SILVA, D. H. Compostos bioativos e atividade antirradicalar em farinhas de bagaço de uvas de diferentes cultivares desidratadas em liofilizador e em estufa. Brazilian Journal of Food Technology, v. 21, p.1-10, 2018.

BORGES, A. M.; PEREIRA, J.; LUCENA, E. M. P. Caracterização da farinha da banana verde. Ciências e Tecnologia dos Alimentos, Campinas, v.29, n.2, p.333-339, 2009.

BRASIL, Instituto Adolfo Lutz. Métodos físico-químicos para análise de alimentos (4th ed., Volume 1, p.1020). São Paulo: Instituto Adolfo Lutz, 2008.

BRASIL. Agência Nacional de Vigilância Sanitária. Resolução n²63, de 2005. Aprova o Regulamento Técnico para Produtos de Cereais, Amidos, Farinhas e Farelos. Disponível em: $\langle$ https://www.saude.rj.gov.br/comum/code/MostrarArquivo.php?C=MjIwMw\%2C\%2C . Acesso em: 13 de April de 2019. 
BRASIL. Decreto $\mathrm{n}^{\circ}$ 12.486, de 20 de outubro de 1978. Normas técnicas especiais relativas a alimentos e bebidas. Diário Oficial do Estado de São Paulo, São Paulo, p. 20, 21 out. 1978.

BRASIL. Ministério da Agricultura, Pecuária e Abastecimento. Instrução normativa n. 62 de 27 de agosto de 2003. Métodos analíticos oficiais para análises microbiológicas para controle de produtos de origem animal e água. Diário Oficial da União, n.181, 18 set. 2003. Seção 1, p.14-51

CAMPOS, R. P.; SILVA, M. J. F.; SILVA, C. F.; FRAGOSO, M. R.; CANDIDO, C. J. Elaboração e Caracterização de Farinha da Casca de Pequi. Cadernos de Agroecologia, v.11, n.2, p.1-12, 2016.

CHISTÉ, R. C.; COHEN, K. O.; MATHIAS, E. A.; RAMOA JUNIOR, A. G. A. Qualidade da farinha de mandioca do grupo seca. Ciências e Tecnologia dos Alimentos, Campinas, v.26, n.4, p.861-864, 2006.

COSTA, C. F.; CORREAA, P. C.; VANEGAS, J. D. B.; BAPTESTINI, F. M.; CAMPOS, R. C.; FERNANDES, L. S. Mathematical modeling and determination of thermodynamic properties of jabuticaba peel during the drying process. Revista Brasileira de Engenharia Agrícola e Ambiental, v.20, n.6, p.576-580, 2016.

FAO. Food and Agriculture Organization of the United Nations. Food Energy: Methods of Analysis and Conversion Factors. Report of a Technical Workshop; Food and Nutrition Paper Volume 77; FAO: Rome, Italy, 2003.

FELLOWS, P. J. Food Processing Technology: Principles and Practice. 1 ed. Porto Alegre, Artmed, 602 p, 2006.

GARMUS, T. T.; BEZERRA, J. R. M. V.; RIGO, M.; CORDOVA, K. R. V. Elaboration of cookie with potato skin flour (Solanum tuberosum L.). Revista Brasileira de Tecnologia Agroindustrial, v.3, n.2, p.56-65, 2009. 
GUO, C.; QIAO, J.; ZHANG, S.; REN, X.; LI, M. Purification of polyphenols from kiwi fruit peel extracts using macroporous resins and high-performance liquid chromatography analysis. International Journal of Food Science \& Technology, v.53, n.6, 2018.

LICHTENTHALER, H. K. Cholorophylls and carotenoids: pigments of photosynthetic biomembranes. In: Packer, L., Douce, R. (Eds.). Methods in Enzymology. London, v.148, p.350-382, 1987.

LIMA, J. P.; PORTELA, J. V. F.; MARQUES, L. R.; ALCÂNTARA, M. A.; EL-AOUAR, A. A. Watermelon flour rind in gluten-free cookies. Ciências Rural, v.45, n.9, p.1688-1694, 2015.

LIMA, J. P.; PORTELA, J. V. F.; MARQUES, L.-R.; ALCÂNTARA, M. A.; EL-AOUAR, Â. A. Farinha de entrecasca de melancia em biscoitos sem glúten. Ciência Rural, v.45, n.9, p.1688-1694, 2015.

OLATUNDE, G. O.; HENSHAW, F. O.; IDOWU, M. A.; \& TOMLINS, K. (2015). Quality attributes of sweet potato flour as influenced by variety, pretreatment and drying method. Food Science \& Nutrition, v.4, n.4, p.623-635, 2015.

REBOUÇAS, T. N. H.; VALVERDE, R. M. V.; TEIXEIRA, H. L. Bromatologia da pimenta malagueta in natura e processada em conserva. Horticultura Brasileira, v.31, p.163-165, 2013.

SANTOS, F. S.; FIGUEIRÊDO, R. M. F.; QUEIROZ, A. J. M.; LIMA, T. L. B.; MOREIRA, I. S. Effect of Dehydration Methods on Okra Chemical and Physical Composition. Journal of Agricultural Science, v.11, n.5, p.236-249, 2019c.

SANTOS, N. C.; ALMEIDA, R. L. J.; QUEIROGA, A. P. R.; FLORÊNCIO, I. M. Avaliação microbiológica da farinha desidratada obtida do aproveitamento da casca da jabuticaba (myrciria cauliflora). In: III Encontro Nacional da Agroindústria, Bananeira-PB,2017. Anais... III ENAG - Bananeiras-PB, 2017.

SANTOS, N. C.; BARROS, S. L.; ALMEIDA, R. L. J.; NASCIMENTO, A. P. S.; ALMEIDA, 
R. D. Influence of temperature in the centesimal composition of pitomba shell (Talisia esculenta). Revista Higiene Alimentar, v.33, p.1477-1481, 2019 b.

SANTOS, N. C.; SANTOS, E. R. M.; BARROS, S. L.; ALMEIDA, R. D.; ALMEIDA, R. L. J.; SILVA, V. M. A.; RIBEIRO, V. H. A.; SANTOS, I. A.; PEREIRA, T. S.; NASCIMENTO, A. P. S. Technological use of kiwi (cv. hayward) shell for elaboration of cookie-type biscuits. International Journal of Development Research, v.9, n.7, p.28852-28857, 2019a.

SILVA, S. N.; MATOS, J. D. P.; SILVA, P. B.; COSTA, Z. R. T.; GOMES, J. P.; SILVA, L. P. F. R.; VIEIRA, A. F.; MELO, B. A.; PRIMO, D. M. B.; ALEXANDRE, H. V. Prediction of Mathematical Models of the Drying Kinetics and Physicochemical Quality of the Chili Pepper. Journal of Agricultural Science, v.10, n.12, p.377- 384, 2018.

VASCONCELOS, R. F.; MELO, E. M. M.; CORREIA, A. G. S.; SOUSA, J. S.; SILVA, C. S. B. Incorporation of flour manufactured from goiaba (psidium guajava) pulp processing residue in cookies. Revista Higiene Alimentar, v.33, p.3455-3459, 2019.

WANG, Y.; LI, L.; LIU, H.; ZHAO, T.; MENG, C.; LIU, Z.; LIU, X. Bioactive compounds and in vitro antioxidant activities of peel, flesh and seed powder of kiwi fruit. International Journal of Food Science \& Technology, v.53, n.9, p.2239-2245, 2018. 